\title{
The accuracy of extended-spectrum beta-lactamase detection in Escherichia coli and Klebsiella pneumoniae in South African laboratories using the Vitek 2 Gram-negative susceptibility card AST-N255
}

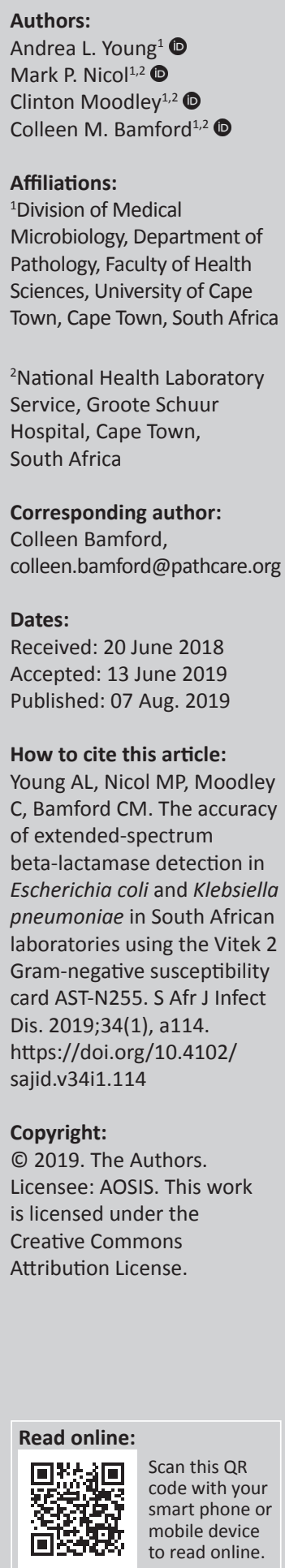

Background: Phenotypic detection of extended-spectrum beta-lactamases (ESBLs) is based on the inhibition of ESBL enzymes by $\beta$-lactamase inhibitors and on the comparison of cephalosporin activity with or without a $\beta$-lactamase inhibitor. Many South African diagnostic laboratories rely on the Vitek 2 for automated susceptibility testing and for ESBL detection. However, the Gram-negative susceptibility card currently used locally (AST-N255) has been modified and its accuracy for ESBL detection is not known.

Methods: We randomly selected 50 isolates of Klebsiella pneumoniae and Escherichia coli from a collection of clinical bloodstream isolates from Groote Schuur Hospital from 2015 to 2016, including ESBL-producing and non-ESBL-producing strains. We used standardised phenotypic (disc diffusion and broth microdilution) and genotypic (conventional polymerase chain reaction (PCR) for $b l a_{C T X-M^{\prime}} b l a_{S H V}$ and $b l a_{T E M}$ ) methods for detection of ESBLs. We compared ESBL detection by Vitek 2 to a composite reference standard comprising ESBL detection either by both phenotypic methods or by one phenotypic method together with genotypic detection.

Results: The sensitivity of Vitek 2 system for detection of ESBLs was 33/36 or 92\% (78\% - 97\%) for E. coli, and $40 / 40$ or $100 \%(91 \%-100 \%)$ for K. pneumoniae, whilst specificity was 10/10 or $100 \%(72 \%-100 \%)$ and $9 / 10$ or $90 \%(60 \%-98 \%)$, respectively. This is comparable with previous studies.

Conclusion: Using a composite reference standard of the phenotypic and genotypic methods employed in this study, no Vitek-categorised ESBL E. coli or K. pneumoniae was found to be a non-ESBL with the exception of possible misinterpretation with K. pneumoniae SHV-hyperproducing isolates.

Keywords: Antimicrobial susceptibility testing; Extended-spectrum beta-lactamase (ESBL) Detection; Automated systems for ESBL Detection; Vitek 2 ESBL detection; Gram-negative susceptibility card AST-N255.

\section{Background}

Extended-spectrum $\beta$-lactamases (ESBLs) are enzymes that hydrolyse most penicillins and cephalosporins, including oxyimino- $\beta$-lactam compounds, but not cephamycins or carbapenems. ${ }^{1}$ Extended-spectrum $\beta$-lactamases are reported to be widespread in South Africa, particularly in Klebsiella pneumoniae ${ }^{2,3}$ and in nosocomial infections. ${ }^{4}$ Rates of ESBL-producing Escherichia coli are also increasing. ${ }^{5}$

As many ESBL-producing organisms are also resistant to other unrelated antibiotics, such as fluoroquinolones, aminoglycosides and trimethoprim-sulfamethoxazole, high rates of ESBL infections result in increased use of carbapenems, which, in turn, selects for carbapenem-resistant organisms, for which there are few therapeutic options.

In routine diagnostic laboratories, phenotypic methods for the detection of ESBLs are based on the inhibition of ESBL enzymes by $\beta$-lactamase inhibitors and on the comparison of cephalosporin activity with or without a $\beta$-lactamase inhibitor. ${ }^{6,7,8}$ This principle underlies both agar-based testing and broth-based testing, including automated susceptibility testing systems such as the Vitek 2 (BioMerieux, Marcy-l'Étoile, France) which is widely used in South Africa. 
However, various modifications to the Gram-negative susceptibility card to meet local needs have necessitated the removal of the cephalosporin-clavulanic acid combination wells. Consequently, ESBL detection in South African laboratories is now based solely on the pattern of susceptibility and resistance to different cephalosporins. Experience with similarly modified Vitek 2 Gram-negative susceptibility cards elsewhere suggests that the specificity of ESBL detection may be reduced. ${ }^{9,10}$ Whilst the Vitek 2 Advanced Expert System ${ }^{\mathrm{TM}}$ (AES), which automatically compares minimum inhibitory concentrations (MICs) of antibiotics to a large database and suggests possible mechanisms of resistance, tends to favour the most conservative options to ensure safe patient treatment, it may thereby add to the over-calling of ESBLs.

The aim of this study was therefore to determine the sensitivity and specificity of the Vitek 2 AST-N255 Gramnegative susceptibility card for the detection of ESBLs in K. pneumoniae and E. coli when compared to standardised phenotypic and genotypic methods.

\section{Methods}

\section{Bacterial isolates}

The Groote Schuur NHLS microbiology laboratory serves the southern part of the greater Cape Town area with a catchment population of approximately 2 million people. The laboratory receives approximately 3600 blood culture samples a month and from these maintains a stored collection of selected organisms. From the 2015-2016 collection of bloodstream isolates, we randomly selected 50 isolates of K. pneumoniae and E. coli, including 40 ESBL-producing and 10 non-ESBLproducing strains of each organism. This categorisation was based on the original identification and susceptibility testing (with Vitek 2 AST-N255 Gram-negative susceptibility card) that was reported in routine diagnostic testing. An isolate was considered an ESBL producer if the phenotypic interpretation by the AES of the Vitek 2 included ESBL with or without decreased outer membrane permeability and not an ESBL if only wild type or $\beta$-lactamases other than ESBLs were suggested by AES. For rapid identification of blood culture isolates, the laboratory uses a previously validated method of direct inoculation from a concentrated suspension of the bottle fluid..$^{11}$ Isolates had been stored as glycerol stocks at $-80^{\circ} \mathrm{C}$ and were subcultured and re-tested with the Vitek 2 to confirm identification and susceptibility test results. The same inoculum was used for concurrent phenotypic and genotypic testing as described below. This second Vitek 2 result was considered the definitive result for comparison purposes. Control strains for ESBL detection, as described below, were tested concurrently.

\section{Phenotypic extended-spectrum beta-lactamase detection}

Extended-spectrum $\beta$-lactamase production was detected by disc diffusion and broth microdilution methods, performed and interpreted according to CLSI criteria. ${ }^{6}$ Appropriate quality control organisms, namely E. coli ATCC ${ }^{(\mathrm{R})} 25922$ and
K. pneumoniae ATCC ${ }^{(\mathrm{R})} 700603$, were included for each method in each run.

Disc diffusion testing was performed using the Kirby-Bauer method. Cefotaxime and ceftazidime discs with or without clavulanic acid were utilised, with an increase of $\geq 5 \mathrm{~mm}$ in zone inhibition diameter for either cephalosporin in the presence of the inhibitor, indicating the presence of an ESBL.

Broth microdilution was performed using the Sensititre ${ }^{\mathrm{TM}}$ ESBL plate format (Trek Diagnostic systems, ThermoScientific Waltham, MA, USA) in accordance with the manufacturer's instructions. The Sensititre ${ }^{\mathrm{TM}}$ ESBL plate includes wells containing cefotaxime and ceftazidime with or without clavulanic acid. $\mathrm{A} \geq 3$ twofold concentration decrease in MIC for either cephalosporin in the presence of the inhibitor indicates the presence of an ESBL.

The ranges of MICs (in $\mu \mathrm{g} / \mathrm{mL}$ ) that can be determined using the Sensititre ESBL plate are $\leq 0.25->64, \leq 0.12 / 4->64 / 4$, $\leq 0.25->128$ and $\leq 0.12 / 4->128 / 4$ for cefotaxime, cefotaxime + clavulanic acid, ceftazidime and ceftazidime + clavulanic acid, respectively. An indeterminate result was reported when it was not possible to calculate the ratio accurately at the limits of MIC range, for example if cefotaxime $\mathrm{MIC} \leq 0.25$, and cefotaxime + clavulanic acid MIC $\leq 0.12 / 4$. If an indeterminate result was obtained for either cefotaxime or ceftazidime, the isolate was categorised according to the result of the other antibiotic. If indeterminate results were obtained for both antibiotics, the ESBL status was determined by the MICs of the antibiotics, that is, if both cefotaxime and ceftazidime MICs were at the lower limit of the MIC range, the isolate was reported as ESBL negative, whereas if both MICs were at the upper limit of the range, the isolate was reported as ESBL-positive.

\section{Genotypic detection of extended-spectrum beta-lactamases}

Bacterial DNA was extracted from colonies grown on $2 \%$ blood agar using the QiaSymphony SP automated extraction platform with the QiaSymphony DSP Virus/Bacteria mini kit, according to the manufacturer's instructions (Qiagen, Hilden, Germany). To test for the presence of the most commonly occurring ESBL genes bla $a_{\mathrm{CTX}-\mathrm{M}^{\prime}} b l a_{\mathrm{SHV}}$ and $b l a_{\mathrm{TEM}^{\prime}}$ conventional PCR assays using primers designed to target internal fragments of these genes were performed. Details of the primer sequences, expected amplicon sizes and amplification conditions are given in the Appendix. The previously described positive and negative controls were included in each run. Selected amplicons including any discrepant genotypic-phenotypic results were submitted for DNA sequencing (Inqaba Biotech, Muckleneuk, Pretoria, South Africa) and the data analysed to confirm the gene identity and genotype, where possible.

\section{Statistical analysis}

The sensitivity and specificity of the Vitek 2 AST-N255 Gram-negative susceptibility card for ESBL detection was 
compared to a composite reference standard, in which an isolate was defined as an ESBL if either an ESBL was detected by both phenotypic methods, that is, by disc diffusion and by broth microdilution, or if an ESBL was detected by either phenotypic method as well as genotypically. The $95 \%$ confidence intervals for proportions were calculated according to Newcombe method. ${ }^{12}$

\section{Ethical consideration}

Ethical clearance was obtained from the Faculty of Health Sciences Human Research Ethics Committee (HREC) (HREC REF: 909/2015).

\section{Results}

We tested 96 isolates including 46 E. coli and 50 K. pneumoniae isolates. Of the $46 \mathrm{E}$. coli isolates, 33 were ESBL producers and 13 non-ESBL producers, according to the definitive Vitek 2 test results, whilst among the K. pneumoniae isolates tested, there were 41 ESBL producers and 9 non-ESBL producers.

\section{Genotypic detection of extended-spectrum beta-lactamase genes $\boldsymbol{b} / \boldsymbol{a}_{\mathrm{CTX}-\mathrm{M}}, \boldsymbol{b} / \boldsymbol{a}_{\mathrm{SHV}}$ and $\boldsymbol{b} / \boldsymbol{a}_{\mathrm{TEM}}$}

Among the $46 \mathrm{E}$. coli isolates tested, $b l a_{\mathrm{CTX}-\mathrm{M}}$ and $b l a_{\mathrm{TEM}}$ were detected in 23 and 13 isolates, respectively. Five isolates contained both $b l a_{\mathrm{CTX}-\mathrm{M}}$ and $b l a_{\mathrm{TEM}^{\prime}}$ whilst $b l a_{\mathrm{SHV}}$ was not detected in any isolate. Among the $50 \mathrm{~K}$. pneumoniae isolates tested, $b l a_{\mathrm{SHV}}$ was detected in all, with 11 isolates containing no other bla gene. The remaining 39 isolates all contained $b l a_{\mathrm{CTX}-\mathrm{M}}$ and 27 also contained $b l a_{\mathrm{TEM}}$.

Sequence analysis of a limited number of gene products confirmed the identification of the ESBL-encoding gene $b l a_{\text {СТХ-M-15 }}$ in four bla $a_{\text {СТХ-M }}$ amplicons in E. coli and in two bla ${ }_{\text {СТХ-M }}$ amplicons in K. pneumoniae. All bla $a_{\mathrm{SHV}}$ and bla ${ }_{\mathrm{TEM}}$ amplicons sequenced were identified as narrow-spectrum betalactamases in both E. coli (7 bla $a_{\text {TEM-1 }}$ isolates) and in $K$. pneumoniae (5 bla $a_{\mathrm{SHv}-1,} 1 b l a_{\mathrm{LEN}-17}, 1 b l a_{\mathrm{LEN}-19}$ and $\left.1 b l a_{\mathrm{TEM}-1}\right)$. No other amplicons were sequenced.

\section{Phenotypic detection of extended-spectrum beta-lactamases}

All isolates were tested for ESBL production using both phenotypic methods. Using the Sensititre method, an indeterminate result was obtained with both cefotaxime and ceftazidime for $10 \mathrm{E}$. coli and $10 \mathrm{~K}$. pneumoniae isolates. These isolates were reclassified according to the MICs of both antibiotics.

\section{Extended-spectrum beta-lactamase detection by Vitek 2 compared to composite reference method}

The composite reference standard comprised either ESBL detection by both phenotypic methods or by a combination of one phenotypic method together with genotypic detection. Given the detection of non-ESBL genes in $b l a_{\mathrm{SHV}}$ and $b l a_{\mathrm{TEM}}$ amplicons, $b l a_{\mathrm{CTX}-\mathrm{M}}$ was the sole target included for genotypic ESBL detection.

The sensitivity of Vitek 2 system for detection of ESBLs as compared to the composite reference standard was $33 / 36$ or $92 \%(78 \%-97 \%)$ for $E$. coli and $40 / 40$ or $100 \%(91 \%-100 \%)$ for K. pneumoniae, whilst specificity was $10 / 10$ or $100 \%$ (72\% - $100 \%)$ and $9 / 10$ or $90 \%(60 \%$ - 98\%), respectively (see Tables 1 and 2).

Detailed analysis of three E. coli and one K. pneumoniae isolates that were misclassified by the Vitek 2 is shown in Appendix Table 1-A1: The only isolate mis-categorised as an ESBL by Vitek 2 was a K. pneumoniae isolate-resistant to ceftazidime only and lacking inhibition by clavulanic acid. The Vitek phenotype listed SHV hyper-production as an alternative resistance phenotype alongside the ESBL resistance phenotype and $b l a_{\mathrm{SHV}}$ was the sole bla gene detected.

Three E. coli isolates classified by the Vitek 2 as having acquired penicilllinases were classified as ESBLs according to the composite reference standard, based on the detection of $b l a_{\text {CTX-M }}$ plus phenotypic detection of an ESBL by one method. The antibiotic susceptibility profile of these isolates according to Vitek 2 showed non-susceptibility to ampicillin, coamoxiclav and cefuroxime and susceptibility to cefotaxime and ceftazidime. The three isolates were also generally susceptible to cefotaxime and ceftazidime by both phenotypic methods with some inconsistent exceptions.

\section{Discussion}

The development of automated susceptibility testing systems and their subsequent introduction into routine diagnostic laboratories sparked an interest in the performance of such systems for the detection of ESBLs and number of studies on the Vitek 2 were conducted, mostly prior to $2010.9,10,13,14,15,16,17,18,19$ These studies vary in many key aspects including the use of different Vitek AST cards, which may $^{10,15,16,17,19}$ or may not ${ }^{9,10,13,14,18}$ contain ESBL confirmatory

TABLE 1: Extended-spectrum beta-lactamase detection by Vitek 2 compared to composite reference method in Escherichia coli.

\begin{tabular}{lccc}
\hline Variable & \multicolumn{3}{c}{ ESBL classification according to composite reference standard } \\
\cline { 2 - 4 } & Positive & Negative & Total \\
\hline Definitive Vitek classification & 33 & 0 & \\
Positive & 3 & 10 & 33 \\
Negative & 36 & 10 & 13 \\
\hline Total & & & 46
\end{tabular}

ESBL, extended-spectrum beta-lactamase.

TABLE 2: Extended-spectrum beta-lactamase detection by Vitek 2 compared to composite reference method in Klebsiella pneumoniae.

Variable ESBL classification according to composite reference standard

\begin{tabular}{lccc} 
& Positive & Negative & Total \\
\hline Definitive Vitek classification & 40 & & \\
Positive & 0 & 1 & 41 \\
Negative & 40 & 10 & 9 \\
\hline Total & & 9 & 50
\end{tabular}

ESBL, extended-spectrum beta-lactamase. 
wells with combinations of cephalosporins and clavulanic acid. Different versions of the Vitek AES software were also used, especially in some of the earlier studies. ${ }^{10,15}$ The organisms tested varied with some studies restricted to $E$. coli and Klebsiella $a^{13,15,17,18}$ as these were the organisms for which phenotypic ESBL testing was recommended by CLSI, whilst others included a wider variety of Enterobacteriaceae including AmpC producers..$^{9,14,19}$ Some studies focussed on clinical isolates, ${ }^{10,13,14,17,18,19}$ whilst others used isolates from large collections specially selected to represent a diversity of resistance mechanisms. ${ }^{15,16}$ Some studies only included isolates presumed to be ESBLs based on screening criteria, ${ }^{10,18}$ whilst the reference methods used for comparison also varied. Overall, sensitivity ranged from $78.0 \%$ to $98.1 \%$, whilst specificity showed greater variation, from as low as $33.3 \%$ to $99.7 \%$. In general, performance was better with Vitek AST cards containing confirmatory wells, 10,15,16,17,19 and when restricted to E. coli and Klebsiella..$^{10,15,16,17}$ In a number of studies, performance was inferior to combination disc testing ${ }^{9,19}$ although other studies suggested that Vitek 2 was adequate for use in routine diagnostic laboratories. ${ }^{14,16,17,19}$ Studies of other automated systems show similar variability in design and limited performance in general.

In this study, the performance of the Vitek 2 AST-N255 card was comparable to previous studies with sensitivities of $92 \%(78 \%-97 \%)$ and $100 \%(91 \%-100 \%)$ and specificities of $100 \%(72 \%-100 \%)$ and $90 \%(60 \%-98 \%)$ for E. coli and $K$. pneumoniae, respectively, against the composite reference standard. The small number of non-ESBL isolates included in this study meant that confidence intervals for all specificity estimates were relatively wide, ranging between of $60 \%$ and $100 \%$.

Limitations of this study included the fact that testing was not performed in duplicate and that the commercial broth microdilution method chosen contained a limited range of antibiotic concentrations which generated many indeterminate results. Genotypic testing was limited to the commonest ESBLs, in particular to the bla $a_{\text {СТХ-M-1 }}$ family of ESBLs and particularly among $E$. coli other types of ESBLs may not have been detected. Genotypic detection of resistance genes does not necessarily correlate with expression, and this, together with slight technical errors in phenotypic testing, may have resulted in the mis-categorisation of three phenotypically nonESBL E. coli isolates as potential ESBLs. Isolates were selected at random from stored clinical isolates, but as this was a single site study, these isolates may not be representative of resistance genotypes present in other geographic areas. The findings are applicable only to the Vitek AST-N255 card or cards with a similar configuration of cephalosporin and cephalosporinclavulanic acid combination wells.

However, using a composite reference standard encompassing combinations of the phenotypic and genotypic methods employed in this study, no Vitek-categorised ESBL E. coli or K. pneumoniae was found to be a non-ESBL with the exception of possible misinterpretation with K. pneumoniae SHV-hyperproducing isolates. $^{20}$

\section{Acknowledgement Competing interest}

The authors have declared that no competing interests exist.

\section{Author contributions}

All authors contributed equally to this work.

\section{Funding}

The authors received funding from the National Health Laboratory Service Research Trust (grant 94570) and the Federation of Infectious Diseases Societies of Southern Africa (FIDSSA)-GlaxoSmithKline Research Fellowship for Antimicrobial Resistance 2015 (no grant number provided) to carry out this research. Neither of the sponsors had any input into the design, performance or reporting of the study.

\section{Data availability statement}

Data sharing is not applicable to this article as no new data were created or analysed in this study.

\section{Disclaimer}

The views and opinions expressed in this article are those of the authors and do not necessarily reflect the official policy or position of any affiliated agency of the authors.

\section{References}

1. Paterson D, Bonomo R. Extended-spectrum-lactamases: A clinical update. Clin Microbiol Rev. 2005;18:657-686. https://doi.org/10.1128/CMR.18.4.657-686.2005

2. Bamford C, Bonorchis K, Elliott E, et al. Antimicrobial susceptibility patterns of selected bacteraemic isolates from public sector hospitals in South Africa, 2010. S Afr J Epidemiol Infect. 2011;26:243-250. https://doi.org/10.1080/10158782.201 Afr J Epidemiol

3. Perovic $O$, Singh-Moodley A, Dusé $A$, et al. National sentinel site surveillance for antimicrobial resistance in Klebsiella pneumoniae isolates in South Africa, 2010-2012. S Afr Med J. 2014;104:563-568. https://doi.org/10.7196/samj.7617

4. McKay R, Bamford C. Community-versus healthcare-acquired bloodstream infections at Groote Schuur Hospital, Cape Town. S Afr Med J. 2015;105:363-369. https://doi.org/10.7196/samj.8183

5. Bamford C, Bonorchis K, Ryan A, et al. Antimicrobial susceptibility patterns of Escherichia coli strains isolated from urine samples in South Africa from 2007-2011. S Afr J Epidemiol Infect. 2012;27(2):46-52. https://doi.org/10.1080/ 10158782.2012.11441483

6. Clinical and Laboratory Standards Institute. Performance standards for antimicrobial susceptibility testing; twenty-sixth informational supplement. Wayne, Pennsylvania: CLSI; 2016.

7. Leclercq R, Canton R, Brown DF, et al. EUCAST expert rules in antimicrobia susceptibility testing. Clin Microbiol Infect. 2013;19:141-160. https://doi.org/ 10.1111/j.1469-0691.2011.03703.x

8. European Committee on Antimicrobial Susceptibility Testing. EUCAST guidelines for detection of resistance mechanisms and specific resistances of clinical and/or epidemiological importance [homepage on the Internet]. Version 1.0. European Committee on Antimicrobial Susceptibility Testing; 2013 [cited 2019 Jul 10] Available from: http://www.eucast.org/resistance_mechanisms/

9. Wiegand I, Geiss H, Mack D, et al. Detection of extended-spectrum betalactamases among enterobacteriaceae by use of semiautomated microbiology systems and manual detection procedures. J Clinl Microbiol. 2007;45:1167-1174. https://doi.org/10.1128/JCM.01988-06

10. Farber J, Moder K-A, Layer F, et al. Extended-spectrum beta-lactamase detection with different panels for automated susceptibility testing and with a chromogenic medium J Clin Microbiol. 2008;46:3721-3727. https://doi.org/10.1128/JCM.00777-08

11. Bamford CM, Goodway J, Hoffmann, R. Rapid identification and susceptibility testing of gram-negative bacilli from blood cultures using Vitek 2 system. S Afr J Epidemiol Infect. 2010;25:28-31.

12. Newcombe R. Two-sided confidence intervals for the single proportion Comparison of seven methods. Stat Med. 1998;17:857-872. http://www. vassarstats.net/. 
13. Leverstein-Van Hall M, Fluit A, Paauw A, et al. Evaluation of the Etest ESBL and the BD Phoenix, VITEK 1, and VITEK 2 automated instruments for detection of extendedspectrum beta-lactamases in multiresistant Escherichia coli and Klebsiella spp. J Clin
Microbiol. 2002;40:3701-3711. https://doi.org/10.1128/jcm.40.10.3703-3711.2002

14. Spanu T, Sanguinetti M, Tumbarello $M$, et al. Evaluation of the new VITEK 2 extended-spectrum beta-lactamase (ESBL) test for rapid detection of ESBL production in enterobacteriaceae isolates. J Clin Microbiol. 2006;44:3257-3262. https://doi.org/10.1128/JCM.00433-06

15. Thomson K, Cornish N, Hong S, et al. Comparison of Phoenix and VITEK 2 extended-spectrum-beta-lactamase detection tests for analysis of Escherichia coli 2007;45:2380-2384. https://doi.org/10.1128/JCM.00776-07

16. Robin F, Delmas J, Schweitzer C, Bonnet R. Evaluation of the Vitek-2 extendedspectrum beta-lactamase test against non-duplicate strains of Enterobacteriacea producing a broad diversity of well-characterised beta-lactamases. Eur J Clin Microbio Infect Dis. 2007;14:148-154. https://doi.org/10.1111/j.1469-0691.2007.01893.x
17. Valenza G, Müller S, Schmitt C, et al. Evaluation of the VITEK 2 AST-N111 card for detection of extended-spectrum beta-lactamases (ESBLs) in Escherichia coli, Klebsiella pneumoniae, and Klebsiella oxytoca compared to ESBL Etests and combination disk methods. Eur J Clin Microbiol. Infect Dis. 2011;30:869-872. https://doi.org/10.1007/s10096-011-1169-2

18. Espinar $M$, Rocha $R$, Ribeiro $M$, et al. Extended-spectrum beta-lactamase of Escherichia coli and Klebsiella pneumoniae screened by the Vitek 2 system. J Med Microbiol. 2011;60:756-760. https://doi.org/10.1099/jmm.0.024075-0

19. Singh R, Singh H. Comparative evaluation of six phenotypic methods for detecting extended-spectrum beta-lactamase-producing Enterobacteriaceae. J Infect Dev Ctries. 2014;8:408-415. https://doi.org/10.3855/jidc.4052

20. Tofteland S, Haldorsen B, Dahl, K, et al. Effects of phenotype and genotype on methods for detection of extended-spectrum- $\beta$-lactamase-producing clinica isolates of Escherichia coli and Klebsiella pneumoniae in Norway. J Clin Microbiol. 2007;45:199-205. https://doi.org/10.1128/JCM.01319-06 


\section{Appendix 1}

Table 1-A1: Analysis of discrepant results for extended-spectrum beta-lactamase detection.

\begin{tabular}{|c|c|c|c|c|c|c|c|c|}
\hline Organism & Isolate & $\begin{array}{l}\text { Resistance phenotype and } \\
\text { susceptibility profile } \dagger \text { according } \\
\text { to Vitek } 2\end{array}$ & $\begin{array}{l}\text { ESBL detection by } \\
\text { disc diffusion }\end{array}$ & $\begin{array}{l}\text { ESBL detection by } \\
\text { Sensititre MIC }\end{array}$ & $\begin{array}{l}\text { Dual phenotypic } \\
\text { methods }\end{array}$ & $\begin{array}{l}\text { CTX-M } \\
\text { detected } \\
\text { by PCR }\end{array}$ & $\begin{array}{l}\text { CTX-M plus } \\
\text { either phenotypic } \\
\text { method }\end{array}$ & $\begin{array}{l}\text { ESBL detection } \\
\text { by composite } \\
\text { reference standard }\end{array}$ \\
\hline E. coli & 8 & $\begin{array}{l}\text { Acquired penicillinase } \\
\text { Non-susceptible to ampicillin, } \\
\text { co-amoxiclav, cefuroxime } \\
\text { Susceptible to cefotaxime, } \\
\text { ceftazidime, cefepime, cefoxitin }\end{array}$ & $\begin{array}{l}\text { Susceptible to } \\
\text { cefotaxime and } \\
\text { ceftazidime }\end{array}$ & $\begin{array}{l}+\S \\
\text { Susceptible to } \\
\text { cefotaxime and } \\
\text { ceftazidime }\end{array}$ & - & + & + & + \\
\hline E. coli & 9 & $\begin{array}{l}\text { Acquired penicillinase } \\
\text { Non-susceptible to ampicillin, } \\
\text { co-amoxiclav, cefuroxime } \\
\text { Susceptible to cefotaxime, } \\
\text { ceftazidime, cefepime, cefoxitin }\end{array}$ & $\begin{array}{l}\text { Susceptible to } \\
\text { cefotaxime and } \\
\text { ceftazidime }\end{array}$ & $\begin{array}{l}+\Phi \\
\text { Susceptible to } \\
\text { cefotaxime } \\
\text { Non-susceptible to } \\
\text { ceftazidime }\end{array}$ & - & + & + & + \\
\hline E. coli & 10 & $\begin{array}{l}\text { Acquired penicillinase } \\
\text { Non-susceptible to ampicillin, } \\
\text { co-amoxiclav, cefuroxime } \\
\text { Susceptible to cefotaxime, } \\
\text { ceftazidime, cefepime, cefoxitin }\end{array}$ & $\begin{array}{l}+\$ \\
\text { Non-susceptible } \\
\text { to cefotaxime } \\
\text { Susceptible to } \\
\text { ceftazidime }\end{array}$ & $\begin{array}{l}\text { Susceptible to } \\
\text { cefotaxime and } \\
\text { ceftazidime }\end{array}$ & + & + & + & + \\
\hline $\begin{array}{l}\text { Klebsiella } \\
\text { pneumoniae }\end{array}$ & 67 & ESBL or SHV hyper-producer & - & + & - & - & - & - \\
\hline
\end{tabular}

$\dagger$, According to CLSI criteria.

$\$$, Cefotaxime zone diameter $(\mathrm{mm})$ : cefotaxime + clavulanic acid zone diameter $(\mathrm{mm})=22: 32$, that is, ESBL-positive; ceftazidime zone diameter $(\mathrm{mm})$ : ceftazidime + clavulanic acid zone diameter $(\mathrm{mm})=26: 27$, that is, ESBL negative.

$\S$, Cefotaxime MIC $(\mu \mathrm{g} / \mathrm{mL})$ : cefotaxime + clavulanic acid MIC $(\mu \mathrm{g} / \mathrm{mL})=1: \leq 0.12 / 4$, that is, ESBL-positive; ceftazidime MIC $(\mu \mathrm{g} / \mathrm{mL}):$ ceftazidime $+\mathrm{clavulanic}$ acid $\mathrm{MIC}(\mu \mathrm{g} / \mathrm{mL})=0.5: \leq 0.12 / 4$, that is, indeterminate.

T, Cefotaxime MIC $(\mu \mathrm{g} / \mathrm{mL})$ : cefotaxime + clavulanic acid MIC $(\mu \mathrm{g} / \mathrm{mL})=0.5: \leq 0.12 / 4$, that is, indeterminate; ceftazidime $\mathrm{MIC}(\mu \mathrm{g} / \mathrm{mL}):$ ceftazidime $+\mathrm{clavulanic}$ acid $\mathrm{MIC}(\mu \mathrm{g} / \mathrm{mL})=16: 0.5 / 4$, that is, ESBL-positive.

$\dagger$, Cefotaxime MIC $(\mu \mathrm{g} / \mathrm{mL})$ : cefotaxime + clavulanic acid MIC $(\mu \mathrm{g} / \mathrm{mL})=1: \leq 0.12 / 4$, that is, ESBL-positive; ceftazidime MIC $(\mu \mathrm{g} / \mathrm{mL}):$ ceftazidime + clavulanic acid MIC $(\mu \mathrm{g} / \mathrm{mL})=0.5: \leq 0.12 / 4$, that is, indeterminate. 